Document downloaded from:

http://hdl.handle.net/10251/52825

This paper must be cited as:

Liu, X.; Wu, S.; Benítez López, J. (2011). On nonsingularity of combinations of two group invertible matrices and two tripotent matrices. Linear and Multilinear Algebra. 59(12):14091417. doi:10.1080/03081087.2011.558843.

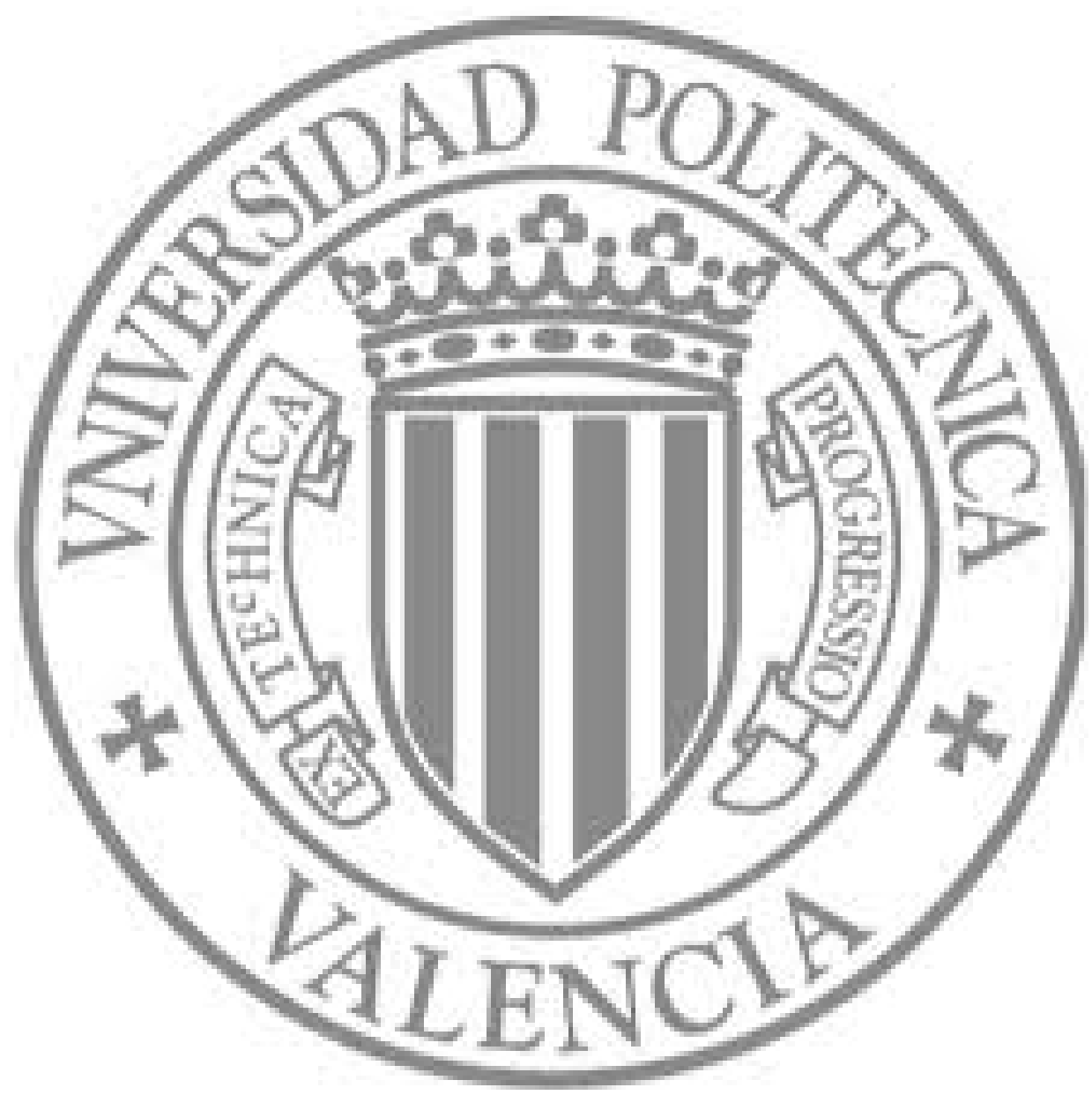

The final publication is available at

http://dx.doi.org/10.1080/03081087.2011.558843

Copyright Taylor \& Francis 


\title{
On nonsingularity of combinations of two group invertible matrices and two tripotent matrices
}

\author{
Xiaoji Liu $^{1} \quad$ Shuxia $\mathrm{Wu}^{1} \quad$ Julio Benítez ${ }^{2 *}$ \\ ${ }^{1}$ College of Mathematics and Computer Science, \\ Guangxi University for Nationalities, Nanning 530006, P.R. China \\ 2 Departamento de Matemática Aplicada, Instituto de matemática \\ multidisciplinar, Universidad Politécnica de Valencia, Valencia 46022, Spain.
}

\begin{abstract}
Let $T_{1}$ and $T_{2}$ be two $n \times n$ tripotent matrices and $c_{1}, c_{2}$ two nonzero complex numbers. We mainly study the nonsingularity of combinations $T=c_{1} T_{1}+c_{2} T_{2}-$ $c_{3} T_{1} T_{2}$ of two tripotent matrices $T_{1}$ and $T_{2}$, and give some formulae for the inverse of $c_{1} T_{1}+c_{2} T_{2}-c_{3} T_{1} T_{2}$ under some conditions. Some of these results are given in terms of group invertible matrices.
\end{abstract}

Key words: Tripotent matrix, Linear combination, Diagonalization, Nonsingularity, Group invertible matrix.

\section{Introduction}

Let $\mathbb{C}$ be the field of complex numbers, and let the symbols $\mathbb{C}^{*}$ and $\mathbb{C}^{n \times n}$ denote the set of nonzero complex numbers and $n \times n$ complex matrices respectively. Moreover, $\mathcal{R}(A), \mathcal{N}(A)$ and $A^{*}$ stand for the column space, null space and conjugate transpose of $A \in \mathbb{C}^{n \times n}$, respectively. A matrix $A \in \mathbb{C}^{n \times n}$ is said to be idempotent if $A^{2}=A$, and tripotent if $A^{3}=A$.

Recall that $A \in \mathbb{C}^{n \times n}$ is nonsingular if and only if $\mathcal{N}(A)=\{0\}$. Also notice that if $T \in \mathbb{C}^{n \times n}$ and $k$ is a natural number greater than 1 , then $T$ satisfies $T^{k}=T$ if and only if $T$ is diagonalizable and the spectrum of $T$ is contained in $\sqrt[k-1]{1} \cup 0$, which have been proved in [5].

Recently, the nonsingularity of linear combinations of idempotent matrices, projectors, tripotent matrices and $k$-potent matrices (see, for example, $[1,2,4,6,7]$ ) have

\footnotetext{
${ }^{*}$ Corresponding author.

E-mail: xiaojiliu72@yahoo.com.cn (X. Liu, Tel. +86-0771-3264782), anita623482950@yahoo.com.cn (S. Wu), jbenitez@mat.upv.es (J. Benítez).

The first orthor was supported by the National Natural Science Foundation of China (11061005) and the Ministry of Education Science and Technology Key Project (210164). The third author has been supported by Spanish Project MTM2010-18539.
} 
been extensively investigated. In [9], Sarduvan and Özdemir have considered the nonsingularity of linear combinations $T=c_{1} T_{1}+c_{2} T_{2}$, where $T_{1}, T_{2}$ are two commuting $n \times n$ tripotent matrices and $c_{1}, c_{2} \in \mathbb{C}^{*}$. In [10], Zuo studied the nonsingularity of combinations $c_{1} P+c_{2} Q-c_{3} P Q$ of two idempotent matrices $P$ and $Q$, and the same author generalized the results in [11].

In this paper, we discuss the nonsingularity of combinations $c_{1} T_{1}+c_{2} T_{2}-c_{3} T_{1} T_{2}$ of two tripotent matrices and give some formulae for the inverse of $c_{1} T_{1}+c_{2} T_{2}-c_{3} T_{1} T_{2}$ under some conditions. We point out that the main results of this article are similar to the ones obtained in [10]. Notice that an idempotent matrix is always a tripotent matrix, but a tripotent matrix may not be idempotent. Special types of matrices, such as idempotents, tripotents, etc, are very useful in many contexts and they have been extensively studied in the literature. For example, quadratic forms with idempotent matrices are used extensively in statistical theory. So it is worth to stress and spread these kinds of results.

A matrix $A \in \mathbb{C}^{n \times n}$ is said to be group invertible if there exists $X \in \mathbb{C}^{n \times n}$ such that

$$
A X A=A, \quad X A X=X, \quad A X=X A .
$$

See $[3$, Chapter 4$]$ for more information on this kind of generalized inverse. It can be proved that the set of matrices $X$ satisfying (1.1) is or empty or a singleton and when is a singleton, it is customary to denote its unique element by $A^{\#}$. Also, it is known [8, Exercise 5.10.12] that a matrix $A \in \mathbb{C}^{n \times n}$ is group invertible if and only if there exist nonsingular $S \in \mathbb{C}^{n \times n}, C \in \mathbb{C}^{r \times r}$ such that $A=S(C \oplus 0) S^{-1}$, being $r$ the rank of $A$. In this situation, one has $A^{\#}=S\left(C^{-1} \oplus 0\right) S^{-1}$.

Evidently, if $T$ is a tripotent matrix, then $T$ is group invertible and $T^{\#}=T$. Many of the results given in this paper will be given in terms of group invertible matrices.

\section{Main results}

In [10, Corollary 2.6], it had been proved that $P-Q$ is nonsingular if and only if $a P+b Q-c P Q$ and $I_{n}-P Q$ are nonsingular for any two idempotent matrices $P, Q \in$ $\mathbb{C}^{n \times n}, a, b \in \mathbb{C}^{*}$. In the following theorem, a similar result is established for tripotent matrices.

Theorem 2.1. Let $T_{1}, T_{2} \in \mathbb{C}^{n \times n}$ be two commuting tripotent matrices. Then $T_{1}-T_{2}$ is nonsingular if and only if $I_{n}-T_{1} T_{2}$ and $T_{1}^{2}+\left(I_{n}-T_{1}^{2}\right) T_{2}$ are nonsingular.

Proof. By a suitable simultaneous diagonalization, there exists $S \in \mathbb{C}^{n \times n}$ such that $T_{1}=S \operatorname{diag}\left(\lambda_{1}, \ldots, \lambda_{n}\right) S^{-1}$ and $T_{2}=S \operatorname{diag}\left(\mu_{1}, \ldots, \mu_{n}\right) S^{-1}$ being $\left\{\lambda_{i}\right\}_{i=1}^{n}$ and $\left\{\mu_{i}\right\}_{i=1}^{n}$, the sets of eigenvalues of $T_{1}$ and $T_{2}$, respectively. Observe that $\lambda_{i}, \mu_{j} \in\{-1,0,1\}$ for all $1 \leq i, j \leq n$ since $T_{1}$ and $T_{2}$ are tripotent. Moreover,

$$
\begin{gathered}
T_{1}-T_{2}=S \operatorname{diag}\left(\lambda_{1}-\mu_{1}, \ldots, \lambda_{n}-\mu_{n}\right) S^{-1}, \\
I_{n}-T_{1} T_{2}=S \operatorname{diag}\left(1-\lambda_{1} \mu_{1}, \ldots, 1-\lambda_{n} \mu_{n}\right) S^{-1},
\end{gathered}
$$


and

$$
T_{1}^{2}+\left(I_{n}-T_{1}^{2}\right) T_{2}=S \operatorname{diag}\left(\lambda_{1}^{2}+\left(1-\lambda_{1}^{2}\right) \mu_{1}, \ldots, \lambda_{n}^{2}+\left(1-\lambda_{n}^{2}\right) \mu_{n}\right) S^{-1} .
$$

Assume that $T_{1}-T_{2}$ is nonsingular. Then $\lambda_{i} \neq \mu_{i}$ for all $i \in\{1, \ldots, n\}$. Hence

$$
\left(\lambda_{i}, \mu_{i}\right) \in\{(1,-1),(1,0),(-1,1),(-1,0),(0,1),(0,-1)\} \quad \text { for all } i=1, \ldots, n .
$$

Easily we have that $1-\lambda_{i} \mu_{i} \neq 0$ and $\lambda_{i}^{2}+\left(1-\lambda_{i}^{2}\right) \mu_{i} \neq 0$ for all $1 \leq i \leq n$. Therefore, $I_{n}-T_{1} T_{2}$ and $T_{1}^{2}+\left(I_{n}-T_{1}^{2}\right) T_{2}$ are nonsingular.

Assume that $I_{n}-T_{1} T_{2}$ and $T_{1}^{2}+\left(I_{n}-T_{1}^{2}\right) T_{2}$ are nonsingular. Since $I_{n}-T_{1} T_{2}$ is nonsingular, then $\lambda_{i} \mu_{i} \neq 1$ for all $1 \leq i \leq n$. If $T_{1}-T_{2}$ were singular, then there would exist $j \in\{1, \ldots, n\}$ such that $\lambda_{j}=\mu_{j}$. Having in mind that $\lambda_{j} \mu_{j} \neq 1$, we would get $\lambda_{j}=\mu_{j}=0$. But now, $\lambda_{j}^{2}+\left(1-\lambda_{j}^{2}\right) \mu_{j}=0$, which would yield the singularity of $T_{1}^{2}+\left(I_{n}-T_{1}^{2}\right) T_{2}$.

Remark: Let $p: \mathbb{C}^{2} \rightarrow \mathbb{C}$ be the following complex polynomial:

$$
p(z, w)=a_{1,0} z+a_{2,0} z^{2}+a_{0,1} w+a_{1,1} z w+a_{2,1} z^{2} w+a_{0,2} w^{2}+a_{1,2} z w^{2}+a_{2,2} z^{2} w^{2},
$$

where $a_{i, j}$ are complex numbers. We have

$$
p\left(T_{1}, T_{2}\right)=S \operatorname{diag}\left(p\left(\lambda_{1}, \mu_{1}\right), \ldots, p\left(\lambda_{n}, \mu_{n}\right)\right) S^{-1} .
$$

Now, if $T_{1}^{2}+\left(I_{n}-T_{1}^{2}\right) T_{2}$ were singular, then by (2.1) there would exist $j \in\{1, \ldots, n\}$ such that

$$
\lambda_{j}^{2}+\left(1-\lambda_{j}^{2}\right) \mu_{j}=0 .
$$

This expression is contradicted by $\lambda_{j}= \pm 1$. Therefore, $\lambda_{j}=0$ and by using again (2.3) we get $\mu_{j}=0$. Therefore, $p\left(T_{1}, T_{2}\right)$ is singular because $p(0,0)=0$. Hence we can formulate the following corollary:

Corollary 2.1. Let $T_{1}, T_{2} \in \mathbb{C}^{n \times n}$ be two commuting tripotent matrices. If $I_{n}-T_{1} T_{2}$ is nonsingular and there exists a polynomial as in (2.2) such that $p\left(T_{1}, T_{2}\right)$ is nonsingular, then $T_{1}-T_{2}$ is nonsingular.

We weaken the hypotheses of the commutativity and the tripotency in the following result.

Theorem 2.2. Let $T_{1}, T_{2} \in \mathbb{C}^{n \times n}$ be two group invertible matrices such that $T_{2} T_{1} T_{1}^{\#}=$ $T_{1} T_{1}^{\#} T_{2}$. If $I_{n}-T_{1}^{\#} T_{2}$ is nonsingular and there exists a polynomial $p$ in two noncommuting variables such that $p(0,0)=0$ and $p\left(T_{1}, T_{2}\right)$ is nonsingular, then $T_{1}-T_{2}$ is nonsingular.

Proof. Let $x \in \mathcal{N}\left(T_{1}-T_{2}\right)$, i.e., $T_{1} x=T_{2} x$. Premultiplying by $T_{1} T_{1}^{\#}$ we get

$$
T_{1} x=T_{1} T_{1}^{\#} T_{2} x .
$$


Premultiplying $T_{1} x=T_{2} x$ by $T_{2} T_{1}^{\#}$ and using $T_{2} T_{1} T_{1}^{\#}=T_{1} T_{1}^{\#} T_{2}$ yield $T_{1} T_{1}^{\#} T_{2} x=$ $T_{2} T_{1}^{\#} T_{2} x$; but having in mind that $T_{1} T_{1}^{\#} T_{2} x=T_{1} x=T_{2} x$ we get

$$
T_{2} T_{1}^{\#} T_{2} x=T_{2} x
$$

From (2.4) and (2.5) we have

$$
T_{1}\left(I_{n}-T_{1}^{\#} T_{2}\right) x=0, \quad T_{2}\left(I_{n}-T_{1}^{\#} T_{2}\right) x=0 .
$$

Since $p(0,0)=0$, there exist two polynomials in noncommuting variables, say $p_{1}$ and $p_{2}$ such that $p\left(T_{1}, T_{2}\right)=p_{1}\left(T_{1}, T_{2}\right) T_{1}+p_{2}\left(T_{1}, T_{2}\right) T_{2}$. Thus from (2.6)

$$
\begin{aligned}
p\left(T_{1}, T_{2}\right)\left(I_{n}-T_{1}^{\#} T_{2}\right) x & =\left(p_{1}\left(T_{1}, T_{2}\right) T_{1}+p_{2}\left(T_{1}, T_{2}\right) T_{2}\right)\left(I_{n}-T_{1}^{\#} T_{1}\right) x \\
& =p_{1}\left(T_{1}, T_{2}\right) T_{1}\left(I_{n}-T_{1}^{\#} T_{2}\right) x+p_{2}\left(T_{1}, T_{2}\right) T_{2}\left(I_{n}-T_{1}^{\#} T_{2}\right) x \\
& =0 .
\end{aligned}
$$

Under the assumption that $I_{n}-T_{1}^{\#} T_{2}$ and $p\left(T_{1}, T_{2}\right)$ are nonsingular, the above computation yields $x=0$, which means that $T_{1}-T_{2}$ is nonsingular.

Remark. Let $T_{1}, T_{2} \in \mathbb{C}^{n \times n}$ be group invertible and $r$ the rank of $T_{1}$. If $T_{1} T_{2}=T_{2} T_{1}$, then by writing $T_{1}=S(C \oplus 0) S^{-1}$, where $S \in \mathbb{C}^{n \times n}$ and $C \in \mathbb{C}^{r \times r}$ are nonsingular we get that $T_{2}$ can be written as $T_{2}=S(D \oplus E) S^{-1}$, where $C D=D C$ and $D \in \mathbb{C}^{r \times r}$. Hence, $T_{1} T_{1}^{\#} T_{2}=S(D \oplus 0) S^{-1}=T_{2} T_{1} T_{1}^{\#}$. However, observe that the condition $T_{1} T_{1}^{\#} T_{2}=T_{2} T_{1} T_{1}^{\#}$ is more general than $T_{1} T_{2}=T_{2} T_{1}$ (it is enough to consider the case when $T_{1}$ is nonsingular and $T_{1} T_{2} \neq T_{2} T_{1}$ ).

Assume in this paragraph that $T_{1}, T_{2} \in \mathbb{C}^{n \times n}$ are two commuting group invertible matrices. Now, if we use the condition $T_{1}^{2} T_{2}=T_{2}^{2} T_{1}$, then we can give some kind of the converse of Theorem 2.2. From $T_{1}^{2} T_{2}=T_{2}^{2} T_{1}$ and $T_{1} T_{2}=T_{2} T_{1}$, we have $\left(T_{1}-T_{2}\right) T_{1} T_{2}=$ 0 , hence the invertibility of $T_{1}-T_{2}$ leads to $T_{1} T_{2}=0$. Thus, $c_{1} T_{1}+c_{2} T_{2}-c_{3} T_{1} T_{2}=$ $c_{1} T_{1}+c_{2} T_{2}$, and we will give the explicit expression of $\left(c_{1} T_{1}+c_{2} T_{2}\right)^{-1}$ in terms of $\left(T_{1}-T_{2}\right)^{-1}$ under mild conditions.

Theorem 2.3. Let $T_{1}, T_{2} \in \mathbb{C}^{n \times n}$ be two group invertible matrices and $c_{1}, c_{2} \in \mathbb{C}^{*}$. If $T_{2} T_{1}=0$ and $T_{1}-T_{2}$ is nonsingular. Then $c_{1} T_{1}+c_{2} T_{2}$ is nonsingular and

$$
\left(c_{1} T_{1}+c_{2} T_{2}\right)^{-1}=\left[\left(c_{1}^{-1}+c_{2}^{-1}\right) T_{1} T_{1}^{\#}-c_{2}^{-1} I_{n}\right]\left(T_{1}-T_{2}\right)^{-1} .
$$

Proof. It follows from the following computations:

$$
\begin{aligned}
& \left(c_{1} T_{1}+c_{2} T_{2}\right)\left[\left(c_{1}^{-1}+c_{2}^{-1}\right) T_{1} T_{1}^{\#}-c_{2}^{-1} I_{n}\right]= \\
& \quad=\left(1+c_{1} c_{2}^{-1}\right) T_{1}-c_{1} c_{2}^{-1} T_{1}+\left(c_{2} c_{1}^{-1}+1\right) T_{2} T_{1} T_{1}^{\#}-T_{2}=T_{1}-T_{2} .
\end{aligned}
$$


Remark. In Theorem 2.3, the situation $c_{1}+c_{2}=0$ is trivial and it does not need the hypothesis $T_{2} T_{1}=0$.

In [10], it was showed that for two idempotent matrices $P, Q \in \mathbb{C}^{n \times n}$, and $a, b \in \mathbb{C}^{*}$, one has that matrix $a P+b Q-(a+b) P Q$ is nonsingular if and only if $\mathbb{C}^{n}=\mathcal{R}\left(P\left(I_{n}-\right.\right.$ $Q)) \oplus \mathcal{R}\left(\left(I_{n}-P\right) Q\right)$ if and only if $\mathbb{C}^{n}=\mathcal{N}\left(P\left(I_{n}-Q\right)\right) \oplus \mathcal{N}\left(\left(I_{n}-P\right) Q\right)$. We give similar results in the following theorem.

Theorem 2.4. Let $T_{1}, T_{2} \in \mathbb{C}^{n \times n}$ be two matrices and $c_{1}, c_{2}, r_{1}, r_{2} \in \mathbb{C}$. If $c_{1} T_{1}+$ $c_{2} T_{2}+\left(r_{1} c_{1}+r_{2} c_{2}\right) T_{1} T_{2}$ is nonsingular, then we have

$$
\mathcal{N}\left[T_{1}\left(I_{n}+r_{1} T_{2}\right)\right] \cap \mathcal{N}\left[\left(I_{n}+r_{2} T_{1}\right) T_{2}\right]=0,
$$

and

$$
\mathcal{R}\left(T_{1}\left(I_{n}+r_{1} T_{2}\right)\right)+\mathcal{R}\left(\left(I_{n}+r_{2} T_{1}\right) T_{2}\right)=\mathbb{C}^{n}
$$

Proof. Denote $\alpha=r_{1} c_{1}+r_{2} c_{2}$. Let $x \in \mathcal{N}\left(T_{1}\left(I_{n}+r_{1} T_{2}\right)\right) \cap \mathcal{N}\left(\left(I_{n}+r_{2} T_{1}\right) T_{2}\right)$. Since $T_{1}\left(I_{n}+r_{1} T_{2}\right) x=0$ and $\left(I_{n}+r_{2} T_{1}\right) T_{2} x=0$, we have

$$
\begin{aligned}
{\left[c_{1} T_{1}+c_{2} T_{2}+\alpha T_{1} T_{2}\right] x } & =\left(c_{1} T_{1}+c_{2} T_{2}+r_{1} c_{1} T_{1} T_{2}+r_{2} c_{2} T_{1} T_{2}\right) x \\
& =c_{1} T_{1}\left(I_{n}+r_{1} T_{2}\right) x+c_{2}\left(I_{n}+r_{2} T_{1}\right) T_{2} x=0 .
\end{aligned}
$$

The nonsingularity of $c_{1} T_{1}+c_{2} T_{2}+\alpha T_{1} T_{2}$ leads to $x=0$. Hence (2.7) holds.

Since $c_{1} T_{1}+c_{2} T_{2}+\alpha T_{1} T_{2}$ is nonsingular, then $\bar{c}_{1} T_{1}^{*}+\bar{c}_{2} T_{2}^{*}+\bar{\alpha} T_{2}^{*} T_{1}^{*}$ is nonsingular. Applying the first part of the proof we have

$$
\mathcal{N}\left(T_{2}^{*}\left(I_{n}+\bar{r}_{2} T_{1}^{*}\right)\right) \cap \mathcal{N}\left(\left(I_{n}+\bar{r}_{1} T_{2}^{*}\right) T_{1}^{*}\right)=0 .
$$

Recalling that $\left[\mathcal{N}\left(X^{*}\right)\right]^{\perp}=\mathcal{R}(X)$ holds for any matrix $X$, by taking perp in (2.9) we get that (2.8) holds.

In the following result, it is given an expression of the inverse of $c_{1} T_{1}+c_{2} T_{2}-c_{3} T_{1} T_{2}$ under some condition.

Theorem 2.5. Let $T_{1}, T_{2} \in \mathbb{C}^{n \times n}$ be two nonzero tripotent matrices such that $T_{1}^{2} T_{2}=$ $T_{2}^{2} T_{1}$ and $c_{1}, c_{2} \in \mathbb{C}^{*}, c_{3} \in \mathbb{C}$. Assume that $T_{1}$ or $T_{2}$ are nonsingular. If $\left(c_{1}+c_{2}\right)^{2}=c_{3}^{2}$, then $c_{1} T_{1}+c_{2} T_{2}-c_{3} T_{1} T_{2}$ or $c_{1} T_{1}+c_{2} T_{2}+c_{3} T_{1} T_{2}$ is singular. If $\left(c_{1}+c_{2}\right)^{2} \neq c_{3}^{2}$, then $c_{1} T_{1}+c_{2} T_{2}-c_{3} T_{1} T_{2}$ is nonsingular; and in this case,

(i) If $T_{1}$ is nonsingular, then

$$
\begin{aligned}
& {\left[\left(c_{1}+c_{2}\right)^{2}-c_{3}^{2}\right]\left(c_{1} T_{1}+c_{2} T_{2}-c_{3} T_{1} T_{2}\right)^{-1} } \\
= & \left(c_{1}+c_{2}\right) T_{1}+c_{3} T_{2}^{2}+c_{1}^{-1} c_{2} c_{3}\left(T_{2}^{2}-T_{1} T_{2}\right)+c_{1}^{-1} c_{3}^{2}\left(T_{2}-T_{1} T_{2}^{2}\right) \\
& +c_{1}^{-1}\left(c_{2}^{2}+c_{1} c_{2}-c_{3}^{2}\right)\left(T_{1}-T_{1} T_{2}^{2}\right) .
\end{aligned}
$$

(ii) If $T_{2}$ is nonsingular, then

$$
\begin{aligned}
& {\left[\left(c_{1}+c_{2}\right)^{2}-c_{3}^{2}\right]\left(c_{1} T_{1}+c_{2} T_{2}-c_{3} T_{1} T_{2}\right)^{-1} } \\
= & \left(c_{1}+c_{2}\right) T_{2}-c_{3}\left(2 T_{1}^{2}-T_{2} T_{1}\right)+c_{2}^{-1}\left(c_{1}^{2}+c_{1} c_{2}-c_{3}^{2}\right)\left(T_{2}-T_{2} T_{1}^{2}\right) .
\end{aligned}
$$


Proof. We split the proof depending if $T_{1}$ or $T_{2}$ is nonsingular.

(i) Let us assume that $T_{1}$ is nonsingular. First, let us prove the first part of the Theorem. The nonsingularity of $T_{1}$ implies $T_{1}^{2}=I_{n}$, hence $T_{1}^{2} T_{2}=T_{2}^{2} T_{1}$ reduces to $T_{2}^{2} T_{1}=T_{2}$. Since $T_{2}$ is tripotent, there exists a nonsingular $S \in \mathbb{C}^{n \times n}$ such that

$$
T_{2}=S\left(\begin{array}{cc}
A & 0 \\
0 & 0
\end{array}\right) S^{-1}, \quad A \in \mathbb{C}^{r \times r}
$$

being $r$ the rank of $T_{2}$. Since $A$ is nonsingular and $T_{2}^{3}=T_{2}$, then $A^{2}=I_{r}$. Let us write

$$
T_{1}=S\left(\begin{array}{cc}
B & C \\
D & E
\end{array}\right) S^{-1}, \quad B \in \mathbb{C}^{r \times r} .
$$

From $T_{2}^{2} T_{1}=T_{2}$ we conclude $B=A$ and $C=0$. Therefore

$$
T_{1}=S\left(\begin{array}{cc}
A & 0 \\
D & E
\end{array}\right) S^{-1}
$$

and

$$
c_{1} T_{1}+c_{2} T_{2}-c_{3} T_{1} T_{2}=S\left(\begin{array}{cc}
\left(c_{1}+c_{2}\right) A-c_{3} I_{r} & 0 \\
c_{1} D-c_{3} D A & c_{1} E
\end{array}\right) S^{-1} .
$$

Since $T_{1}^{2}=I_{n}$, we obtain that $E$ is nonsingular and $E^{2}=I_{n-r}$. From (2.13) we get that $c_{1} T_{1}+c_{2} T_{2}-c_{3} T_{1} T_{2}$ is nonsingular if and only if $\left(c_{1}+c_{2}\right) A-c_{3} I_{r}$ is nonsingular (recall that the first row in the block matrix appearing in (2.13) must be present, since otherwise, $\left.T_{2}=0\right)$.

Since we have

$$
\left[\left(c_{1}+c_{2}\right) A-c_{3} I_{r}\right]\left[\left(c_{1}+c_{2}\right) A+c_{3} I_{r}\right]=\left[\left(c_{1}+c_{2}\right)^{2}-c_{3}^{2}\right] I_{r},
$$

we get that if $\left(c_{1}+c_{2}\right)^{2}-c_{3}^{2}=0$, then $\left(c_{1}+c_{2}\right) A-c_{3} I_{r}$ or $\left(c_{1}+c_{2}\right) A+c_{3} I_{r}$ is singular, which in view of $(2.13)$ we get that $c_{1} T_{1}+c_{2} T_{2}-c_{3} T_{1} T_{2}$ or $c_{1} T_{1}+c_{2} T_{2}+c_{3} T_{1} T_{2}$ is singular.

Now, let us prove the second part of the Theorem, i.e., we shall prove (2.10) for any $c_{1}, c_{2} \in \mathbb{C}^{*}$ satisfying $\left(c_{1}+c_{2}\right)^{2} \neq c_{3}^{2}$. From $(2.14)$ we get that $\left(c_{1}+c_{2}\right)^{2}-c_{3}^{2} \neq 0$ leads to the nonsingularity of $\left(c_{1}+c_{2}\right) A-c_{3} I_{r}$ and

$$
\left[\left(c_{1}+c_{2}\right) A-c_{3} I_{r}\right]^{-1}=\frac{1}{\left(c_{1}+c_{2}\right)^{2}-c_{3}^{2}}\left[\left(c_{1}+c_{2}\right) A+c_{3} I_{r}\right]
$$

Since $T_{1}^{2}=I_{n}$, we have $T_{1}^{-1}=T_{1}$ and from (2.12) we have

$$
T_{1}^{-1}=S\left(\begin{array}{cc}
A & 0 \\
-E D A & E
\end{array}\right) S^{-1}
$$

from them we can conclude that $D=-E D A$, i.e., $-D A=E D$. 
If $c_{1}, c_{2} \in \mathbb{C}^{*}$ satisfy $\left(c_{1}+c_{2}\right)^{2} \neq c_{3}^{2}$, then by using $(2.13)$

$$
\begin{aligned}
& \left(c_{1} T_{1}+c_{2} T_{2}-c_{3} T_{1} T_{2}\right)^{-1} \\
= & S\left(\begin{array}{cc}
{\left[\left(c_{1}+c_{2}\right) A-c_{3} I_{r}\right]^{-1}} & 0 \\
-c_{1}^{-1} E\left(c_{1} D-c_{3} D A\right)\left[\left(c_{1}+c_{2}\right) A-c_{3} I_{r}\right]^{-1} & c_{1}^{-1} E
\end{array}\right) S^{-1} \\
= & S\left(\begin{array}{cc}
{\left[\left(c_{1}+c_{2}\right)^{2}-c_{3}^{2}\right]^{-1}\left[\left(c_{1}+c_{2}\right) A+c_{3} I_{r}\right]} & 0 \\
-c_{1}^{-1} E\left(c_{1} D-c_{3} D A\right)\left[\left(c_{1}+c_{2}\right)^{2}-c_{3}^{2}\right]^{-1}\left[\left(c_{1}+c_{2}\right) A+c_{3} I_{r}\right] & c_{1}^{-1} E
\end{array}\right) S^{-1} .
\end{aligned}
$$

Hence

$$
\begin{aligned}
& {\left[\left(c_{1}+c_{2}\right)^{2}-c_{3}^{2}\right]\left(c_{1} T_{1}+c_{2} T_{2}-c_{3} T_{1} T_{2}\right)^{-1} } \\
= & \left(c_{1}+c_{2}\right) S\left(\begin{array}{cc}
A & 0 \\
-E D A & E
\end{array}\right) S^{-1}+c_{3} S\left(\begin{array}{cc}
I_{r} & 0 \\
0 & 0
\end{array}\right) S^{-1}+c_{1}^{-1} c_{2} c_{3} S\left(\begin{array}{cc}
0 & 0 \\
E D & 0
\end{array}\right) S^{-1} \\
& +c_{1}^{-1} c_{3}^{2} S\left(\begin{array}{cc}
0 & 0 \\
E D A & 0
\end{array}\right) S^{-1}+c_{1}^{-1}\left(c_{2}^{2}+c_{1} c_{2}-c_{3}^{2}\right) S\left(\begin{array}{cc}
0 & 0 \\
0 & E
\end{array}\right) S^{-1} .
\end{aligned}
$$

On the other hand, we have

$$
\begin{gathered}
T_{2}^{2}=S\left(\begin{array}{cc}
I_{r} & 0 \\
0 & 0
\end{array}\right) S^{-1}, \\
T_{2}^{2}-T_{1} T_{2}=S\left(\begin{array}{cc}
0 & 0 \\
E D & 0
\end{array}\right) S^{-1}, \\
T_{2}-T_{1} T_{2}^{2}=S\left(\begin{array}{cc}
0 & 0 \\
E D A & 0
\end{array}\right) S^{-1}, \\
T_{1}-T_{1} T_{2}^{2}=S\left(\begin{array}{cc}
0 & 0 \\
0 & E
\end{array}\right) S^{-1} .
\end{gathered}
$$

From (2.16)-(2.20), we get that (2.10) holds.

(ii) Assume that $T_{2}$ is nonsingular. First, let us prove the first part of the Theorem. As in (i), but now interchanging the roles of $T_{1}$ and $T_{2}$, we can write

$$
T_{1}=S\left(\begin{array}{cc}
A & 0 \\
0 & 0
\end{array}\right) S^{-1}, \quad T_{2}=S\left(\begin{array}{cc}
A & 0 \\
D & E
\end{array}\right) S^{-1}, \quad A \in \mathbb{C}^{r \times r},
$$

being $r$ the rank of $T_{1}$. Since $T_{2}^{2}=I_{n}$ we have $A^{2}=I_{r}$ and $E^{2}=I_{n-r}$. The difference from (i) is

$$
c_{1} T_{1}+c_{2} T_{2} \pm c_{3} T_{1} T_{2}=S\left(\begin{array}{cc}
\left(c_{1}+c_{2}\right) A \pm c_{3} I_{r} & 0 \\
c_{2} D & c_{2} E
\end{array}\right) S^{-1} .
$$

As in (i), if $\left(c_{1}+c_{2}\right)^{2}=c_{3}^{2}$, by (2.14) and (2.21), one has that $c_{1} T_{1}+c_{2} T_{2}+c_{3} T_{1} T_{2}$ or $c_{1} T_{1}+c_{2} T_{2}-c_{3} T_{1} T_{2}$ is singular. 
Now, we shall prove (2.11) for any $c_{1}, c_{2} \in \mathbb{C}^{*}$ satisfying $\left(c_{1}+c_{2}\right)^{2} \neq c_{3}^{2}$. By using (2.21) one has

$$
\begin{aligned}
& \left(c_{1} T_{1}+c_{2} T_{2}-c_{3} T_{1} T_{2}\right)^{-1} \\
= & S\left(\begin{array}{cc}
{\left[\left(c_{1}+c_{2}\right) A-c_{3} I_{r}\right]^{-1}} & 0 \\
-E D\left[\left(c_{1}+c_{2}\right) A-c_{3} I_{r}\right]^{-1} & c_{2}^{-1} E
\end{array}\right) S^{-1} \\
= & S\left(\begin{array}{cc}
{\left[\left(c_{1}+c_{2}\right)^{2}-c_{3}^{2}\right]^{-1}\left[\left(c_{1}+c_{2}\right) A+c_{3} I_{r}\right]} & 0 \\
-E D\left[\left(c_{1}+c_{2}\right)^{2}-c_{3}^{2}\right]^{-1}\left[\left(c_{1}+c_{2}\right) A+c_{3} I_{r}\right] & c_{2}^{-1} E
\end{array}\right) S^{-1} .
\end{aligned}
$$

Hence

$$
\begin{aligned}
& {\left[\left(c_{1}+c_{2}\right)^{2}-c_{3}^{2}\right]\left(c_{1} T_{1}+c_{2} T_{2}-c_{3} T_{1} T_{2}\right)^{-1} } \\
= & S\left(\begin{array}{cc}
\left(c_{1}+c_{2}\right) A+c_{3} I_{r} & 0 \\
-E D\left[\left(c_{1}+c_{2}\right) A+c_{3} I_{r}\right] & c_{2}^{-1}\left[\left(c_{1}+c_{2}\right)^{2}-c_{3}^{2}\right] E
\end{array}\right) S^{-1} \\
= & \left(c_{1}+c_{2}\right) S\left(\begin{array}{cc}
A & 0 \\
-E D A & E
\end{array}\right) S^{-1}+S\left(\begin{array}{cc}
c_{3} I_{r} & 0 \\
-c_{3} E D & c_{2}^{-1}\left(c_{1}^{2}+c_{1} c_{2}-c_{3}^{2}\right) E
\end{array}\right) S^{-1} \\
= & \left(c_{1}+c_{2}\right) T_{2}+c_{3} S\left(\begin{array}{cc}
I_{r} & 0 \\
-E D & 0
\end{array}\right) S^{-1}+c_{2}^{-1}\left(c_{1}^{2}+c_{1} c_{2}-c_{3}^{2}\right) S\left(\begin{array}{cc}
0 & 0 \\
0 & E
\end{array}\right) S^{-1} .
\end{aligned}
$$

On the other hand, we easily have

$$
2 T_{1}^{2}-T_{2} T_{1}=S\left(\begin{array}{cc}
I_{r} & 0 \\
E D & 0
\end{array}\right) S^{-1} \quad \text { and } \quad T_{2}-T_{2} T_{1}^{2}=S\left(\begin{array}{cc}
0 & 0 \\
0 & E
\end{array}\right) S^{-1} .
$$

The proof is completed.

If $c_{3}=0$, then we have the following corollary.

Corollary 2.2. [4, Theorem 3.1] Let $T_{1}, T_{2} \in \mathbb{C}^{n \times n}$ be two nonzero tripotent matrices such that $T_{1}^{2} T_{2}=T_{2}^{2} T_{1}$ and $c_{1}, c_{2} \in \mathbb{C}^{*}$. If $T_{1}$ or $T_{2}$ are nonsingular, then $c_{1} T_{1}+c_{2} T_{2}$ is nonsingular if and only if $c_{1}+c_{2} \neq 0$. In this case,

(i) If $T_{1}$ is nonsingular, then

$$
\left(c_{1}+c_{2}\right)\left(c_{1} T_{1}+c_{2} T_{2}\right)^{-1}=T_{1}+c_{2} c_{1}^{-1} T_{1}\left(I_{n}-T_{2}^{2}\right) .
$$

(ii) If $T_{2}$ is nonsingular, then

$$
\left(c_{1}+c_{2}\right)\left(c_{1} T_{1}+c_{2} T_{2}\right)^{-1}=T_{2}+c_{2} c_{1}^{-1} T_{2}\left(I_{n}-T_{1}^{2}\right) .
$$

The following theorem shows that the nonsingularity of $c_{1} T_{1}+c_{2} T_{2}-c_{3} T_{1} T_{2}$ is also related to the nonsingularity of the same combination of $T_{1}^{2} T_{2}$ and $T_{2}^{2} T_{1}$ or $T_{2} T_{1}^{2}$ and $T_{1} T_{2}^{2}$.

Theorem 2.6. Let $T_{1}, T_{2} \in \mathbb{C}^{n \times n}$ be two tripotent matrices and any $c_{1}, c_{2} \in \mathbb{C}^{*}$. The following statements are equivalent:

(i) $c_{1} T_{2}^{2} T_{1}+c_{2} T_{1}^{2} T_{2}-c_{3} T_{2}^{2} T_{1} T_{2}$ is nonsingular. 
(ii) $c_{1} T_{1} T_{2}^{2}+c_{2} T_{2} T_{1}^{2}-c_{3} T_{1} T_{2} T_{1}^{2}$ is nonsingular.

(iii) $c_{1} T_{1}+c_{2} T_{2}-c_{3} T_{1} T_{2}$ and $I_{n}-T_{1}^{2}-T_{2}^{2}$ are nonsingular.

Proof. The results follow quite easily from the equalities

$$
\left(I_{n}-T_{1}^{2}-T_{2}^{2}\right)\left(c_{1} T_{1}+c_{2} T_{2}-c_{3} T_{1} T_{2}\right)=-\left(c_{1} T_{2}^{2} T_{1}+c_{2} T_{1}^{2} T_{2}-c_{3} T_{2}^{2} T_{1} T_{2}\right)
$$

and

$$
\left(c_{1} T_{1}+c_{2} T_{2}-c_{3} T_{1} T_{2}\right)\left(I_{n}-T_{1}^{2}-T_{2}^{2}\right)=-\left(c_{1} T_{1} T_{2}^{2}+c_{2} T_{2} T_{1}^{2}-c_{3} T_{1} T_{2} T_{1}^{2}\right) .
$$

Acknowledgement: The authors wish to thank the referee for his/her careful review and comments which improved the quality of the paper.

\section{References}

[1] J.K. Baksalary and O.M. Baksalary, Nonsingularity of linear combinations of idempotent matrices, Linear Algebra Appl., 388(2004), 25-29.

[2] J.K. Baksalary, O.M. Baksalary and H. Özdemir, A note on linear combinations of commuting tripotent matrices, Linear Algebra Appl., 388(2004), 45-51.

[3] A. Ben-Israel and T.N.E. Greville, Generalized inverses. Theory and applications. Second edition. CMS Books in Mathematics Springer-Verlag, New York, 2003.

[4] J. Benítez, X. Liu and T. Zhu Nonsingularity and group invertibility of linear combination of two k-potent matrices, Linear Multilinear Algebra, 58(2010), 1023-1035.

[5] J. Benítez and N. Thome, k-Group periodic matrices, SIAM. J. Matrix Anal. Appl., 28(2006), 9-25.

[6] J. Groß and G. Trenkler, Nonsingularity of the difference of two oblique projectors, SIAM J, Matrix Anal. Appl., 21(1999), 390-395.

[7] J.J Koliha, V. Rakočević and I. Straškraba, The difference and sum of projectors, Linear Algebra Appl., 388(2004), 279-288.

[8] C. Meyer, Matrix analysis and applied linear algebra. Society for Industrial and Applied Mathematics (SIAM), Philadelphia, PA, 2000.

[9] M. Sarduvan and H. Özdemir, On nonsingularity of linear combinations of tripotent matrices, (In press)

[10] K. Zuo, Nonsingularity of the difference and the sum of two idempotent matrices, Linear Algebra Appl., 433(2010), 476-482.

[11] K. Zuo and T. Xie, Nonsingularity of combinations of idempotent matrices, J. of Math. (PRC), 29(2009), 285-288. 